\title{
New technologies of human/machine interaction: a prospective study in the military naval context
}

\author{
L André*1, R Coutellier*, C Maï**, A Bonnaud* \\ * Naval Group Research, France \\ ${ }^{I}$ Corresponding Author. Email: rozenn.coutellier@naval-group.com, chantal.mais@ @naval-group.com
}

\begin{abstract}
Synopsis
Today, military defense vessels are equipped with many systems that allow sailors to interact with each other or with their digital equipments. These systems are relatively efficient and allow mariners to perform their tasks efficiently and securely.

It is important to identify new technologies that sailors can interact with, in the future. An evaluation must then be conducted to ensure compliance with their usefulness, usability and acceptability. This paper discusses how to study, upstream, various innovative technologies in order to identify the positive and negative points and to conduct a human factors evaluation following a user-centric approach, replicating operational conditions.

The paper focuses then on three widely available technologies. The first is the eye-control, which allows an operator to interact with a digital system thanks to the movements and fixation of his eyes. This system allows validating information being displayed on a screen or to navigate in an interface when the operator has his hands busy with another task. Different interactions are available today (scrolling, clicking, and displaying a keyboard to write using the eyes ...). However, various limitations were highlighted during the first human factors evaluations, for example visual fatigue or calibration of the eye-tracking system, which is also sensitive to the movements of the operator and those of the platform on which it is based. The second and the third technologies presented are related because they both concern communications. In very noisy environments or when there are different sound sources, it is sometimes difficult for operators to be attentive to all auditory information or to be heard effectively. Bone conduction systems (for listening and for expression) allow the operator to be attentive to different sound sources while speaking audibly. As for the bone conduction listening system, the sound vibrations conducted by the bones reproduce a listening equivalent to classical hearing. Concerning the throat microphone, the treatment of the waves captured at the throat makes it possible to transmit a clear sound, without any environmental interference, which makes it possible to guarantee the good intelligibility of the speech.

This paper concludes on how these studies from the human factor service of the research and development department of Naval Group (France) are related to advance research in these areas as well as trials for future equipment that can be developed on board for naval defense vessels.
\end{abstract}

Keywords: eye-control, new technologies, human factors, bone conduction, throat microphone

\footnotetext{
Author's Biography

Lucile André holds the current position of human factors specialist at Naval Group Research, in France. She is responsible for evaluating the new technologies in a human factor's perspective and her main focus is humansystems interactions. She graduated from Aix-Marseille University in 2019 and holds a degree in psychology with a major in human factors.

Rozenn Coutellier is a Human Factors specialist at Naval Group Research. She is in charge of the installation and operationalization of the Sea U Lab. Her previous experience includes both HMI ergonomics and organizational aspects for user impacts: analysis, design and evaluation in a User Centered Design approach. Chantal Maïs is a human Factors expert. She is in charge of research in this area at Naval Group Research. She is responsible for innovative exploitation with a Human-Centered Design. Her previous experience includes Human-Factor studies in naval ship design.

Aymeric Bonnaud has 30+ years of experience in R\&D on various naval systems within the French Armament Corps, then within Naval Group. He is now director of the Centre of Expertise of the group for Mastering Information engineering, human factors and Signatures (CEMIS). Aymeric Bonnaud is a member of different technical societies in these fields.
} 


\section{Introduction: new human/System interactions (HSI) and challenges in the naval defence field}

Touch screens, keyboards, mouse, headsets, microphones, joysticks..., these are all tools that seem natural today when we talk about human-machine interactions. Everyone has already had the opportunity to use them in different professional or personal contexts in recent decades. Humans are increasingly in contact with machines, in an environment made of technologies. Human activity, based on access to information, knowledge and communication, operates today entirely through new technologies. Within this digital environment, interaction methods have been enriched and increased their technical feasibility. The new human / machine interactions are those of tomorrow, those that have been dreamed of that have not yet succeeded in achieving enough an accessible and simple way of controlling and then need to be more developed.

The naval defence sector is a tense field, due to its obligation to be always at the cutting edge of technology and due to the need for operational performance of sailors and systems. Operational efficiency and usability are of paramount importance in this domain, since at the end of the day sailors' lives are directly linked to the success of the action.. Systems have to protect the human potential of the operators that will be for sure severely engaged in any military action of some intensity.

It is therefore necessary to combine performance and innovation, while guaranteeing to the sailors an ergonomics of the systems, passing by the utility of the services offered, the usability of the systems which they will come to use and ensuring the good acceptability of the innovations to edge. The risk is an operator's lack of interest in the system, followed by a failure in performance, possible errors, loss of time and money.

The main objective of this study was to carry out research in order to explain and to perceive the possibilities of use, the advantages and limits of the new technologies: eye-control, bone conduction headset and throat microphone. The human factors' service of Naval Group Research conducted three pilot studies in order to test these innovative systems, from a point of view of usefulness and usability.

\section{Eye-control: a promising tool?}

\subsection{State of the art}

Eye-control allows people to navigate the interface of a digital system equipped with an eye-tracker (computer, tablet, phone) and to control it simply by looking at it (Rudall \& Mann, 2007). Replacing manual interactions (mouse, keyboard, etc.), the eye-control technology allows, thanks to the tracking of the movements of the eyes, to move the cursor on the screen following the direction of gaze of the user. This also allows, when the user performs an action defined in advance (fixation for a certain period, blinking of the eye, etc.) to replace the effect of a mouse click and therefore to perform actions normally transmitted to the computer through mouse clicks or use of the keyboard. The positive points noted during the analysis of the eye-control systems are the ease of use, because the system requires little handling and reduces load on the hands and arms, which no longer need to be used to interact with the interface (Drewes, 2010). In addition, the use of gaze to navigate an interface is relevant because the users are familiar with the use of their eyes to contextualize their request (for example during a conversation, the speaker will fix his interlocutor in order to show that he is addressing him). Furthermore, Sibert and Jacob (2000) point out that one of the major benefits of using eye control for attentional tasks is that the position of the eye implicitly indicates the area where the user is focusing his attention. However, Ohno (2000) has carried out a study on eye control and highlights a limit which it seems important to consider: visual fatigue can appear quickly when a person tries to focus his gaze on a specific point. Vertegaal (2008) also highlighted the point that humans are not familiar with using their eyes to point to things. Indeed, the primary objective of the eyes is to provide visual feedback for the processing of the environment by the brain, and not to interact with the external environment to perform an action.

\subsection{Methods}

Participants were selected on a voluntary basis, after responding to a questionnaire concerning their profile (age, gender and vision problems). Twelve participants participated, including nine men and three women. The average age of the participants was 29 , the youngest was 23 , the oldest 49 .

This experiment was conducted on an MSI computer, equipped with Windows 10 (interface integrating eyecontrol functionalities, like pointing, clicks, scrolling...) and with the Tobii 4C eye-tracker. Three questionnaires were used: SMEQ evaluation scale, measuring the effort perceived when carrying out a task (Zijlstra, 1985); Long questionnaire to assess visual fatigue (Laharie, 1982) and Short questionnaire to assess visual fatigue (Römer, 1989). 
Participants are welcomed at the laboratory, signed the consent form and filled out the long questionnaire on eyestrain (Laharie, 1982). The calibration of the eye-control is then carried out in order to adapt the system to them. The test takes place in four main timed stages, illustrated on Figure 1. Each step is followed by the completion of the short questionnaire on visual fatigue and of the SMEQ (scale of perception of the effort provided to complete a task).

1. The Beat Shot game (available on the Tobii Games center) was played for one minute. The aim of the game is to target some points with the eyes.

2. Survey: Participants must complete the survey (developed by authors) by simply using their eyes. It included 5 missions: Select an answer from 4; select an image among 3; select 4 boxes among 16; evaluate on a graduated scale and fill a field with a given word.

3. Origami: Participants must, in parallel with the making of an origami, follow the instructions displayed on the screen and scroll the page as they go to fold.

4. Points to connect/drawing: participants must connect the points using their eyes to move the pointer and the mouse to validate the position and connect two points by clicking. This was developed on Axure by the human factors' specialists of Naval Group.

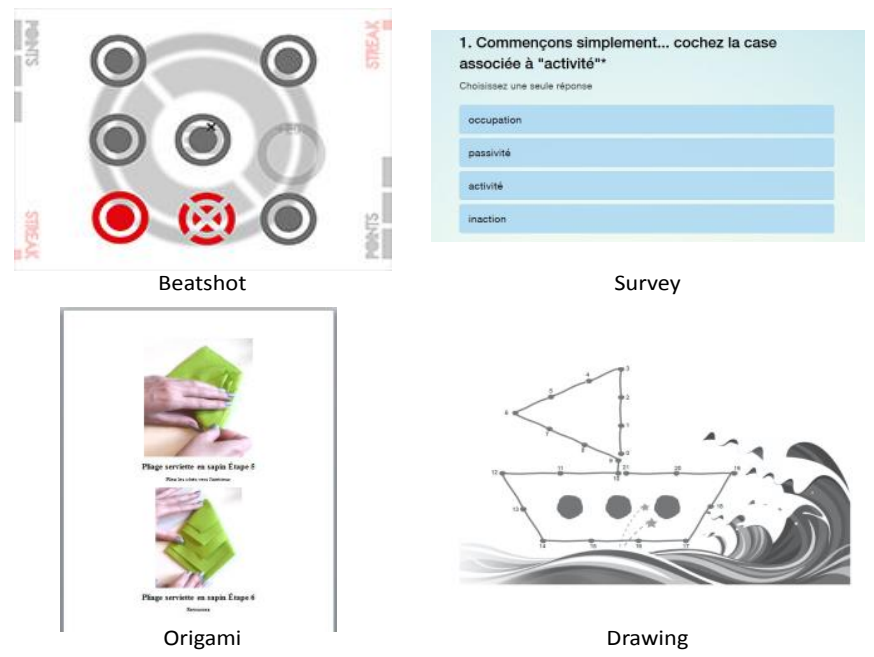

Figure 1 : Illustration of the four stages of the protocol

Once the four steps have been completed, the participant is invited to fill out the long questionnaire on eyestrain again. Then, he must note out of 10 the understanding, the ease of use and the visual fatigue generated by each interaction (fixation, simple click, scrolling of the page, writing on the keyboard, synchronization of eye movements and mouse clicks and general).

\subsection{Results}

Visual fatigue was assessed in two different ways, the results of which will be presented one after the other. First, the long questionnaire on visual fatigue was completed twice, once before and once after the experiment. An increase in fatigue can be seen very clearly on Figure 2.

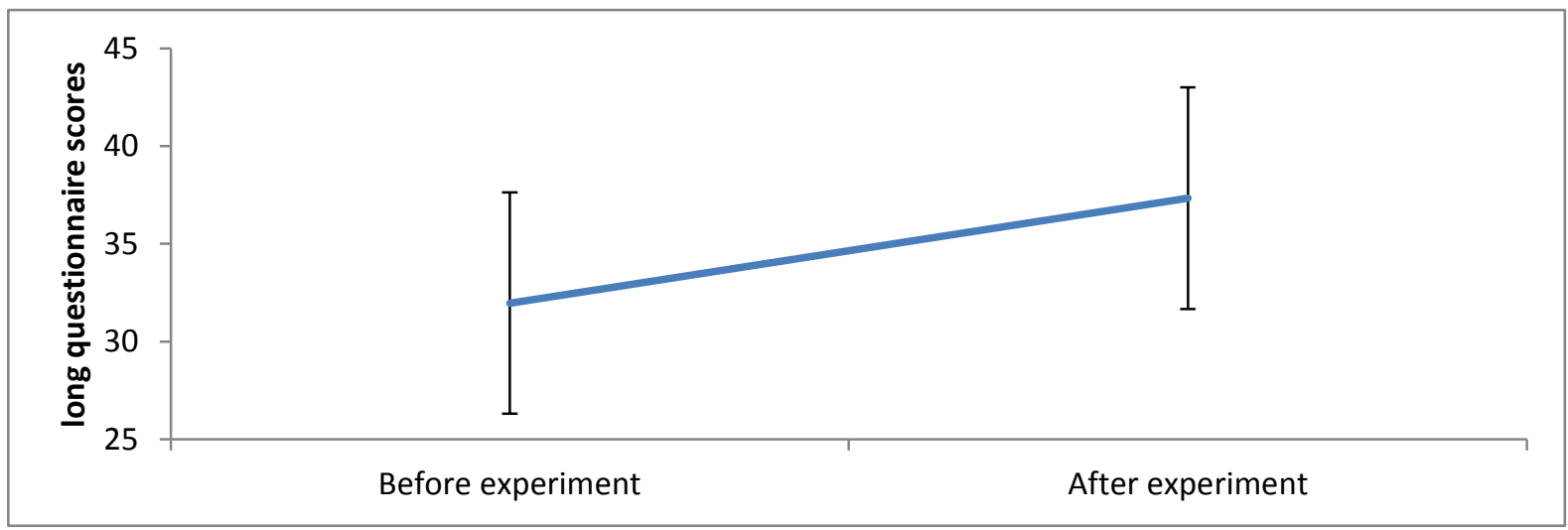

Figure 2 : evolution of long questionnaire scores 
Then, visual fatigue was measured after each step, thanks to the short questionnaire on visual fatigue. If we compare the four points on Figure 3, it is possible to notice that the Beatshot game did not generate much fatigue, that a marked increase of fatigue is visible for the survey part and that the last two stages have was moderately tiring for the participant.

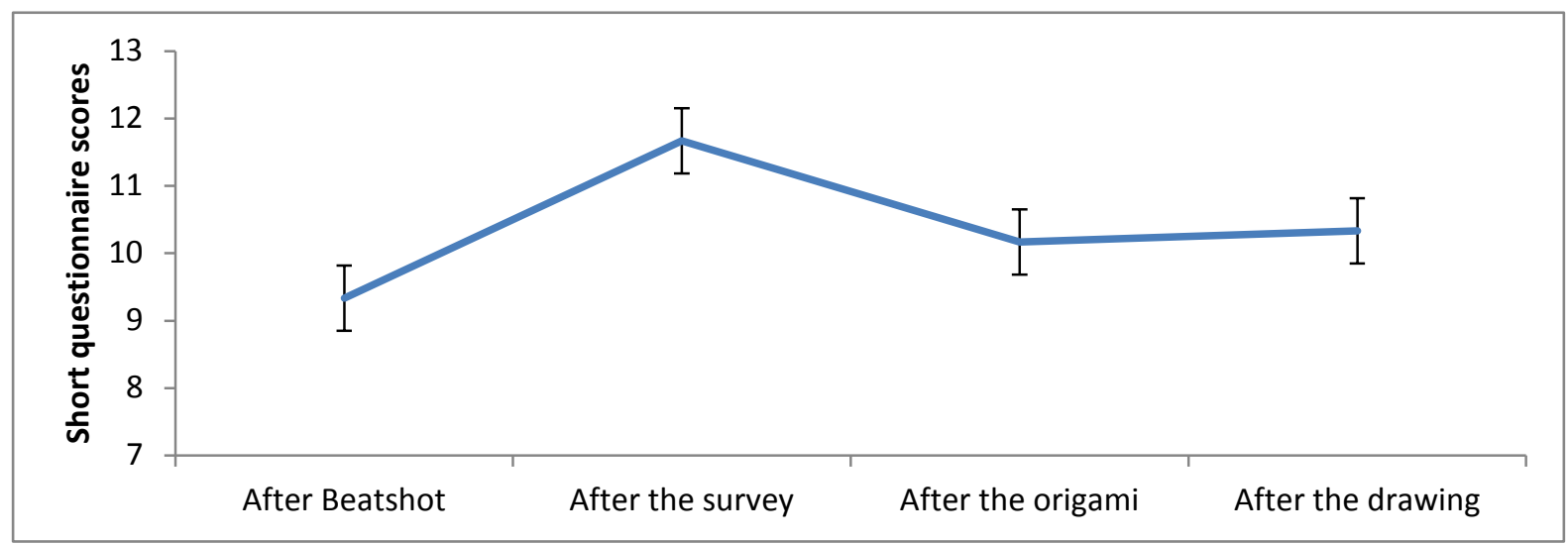

Figure 3 : evolution of short questionnaire scores

Participants were asked to evaluate the ease of use for all interactions they did during the experiment. As shown on Figure 4, participants found it less difficult to type on the keyboard than all other interactions, which were considered moderately easy. Scrolling the page was considered the most complicated task to perform.

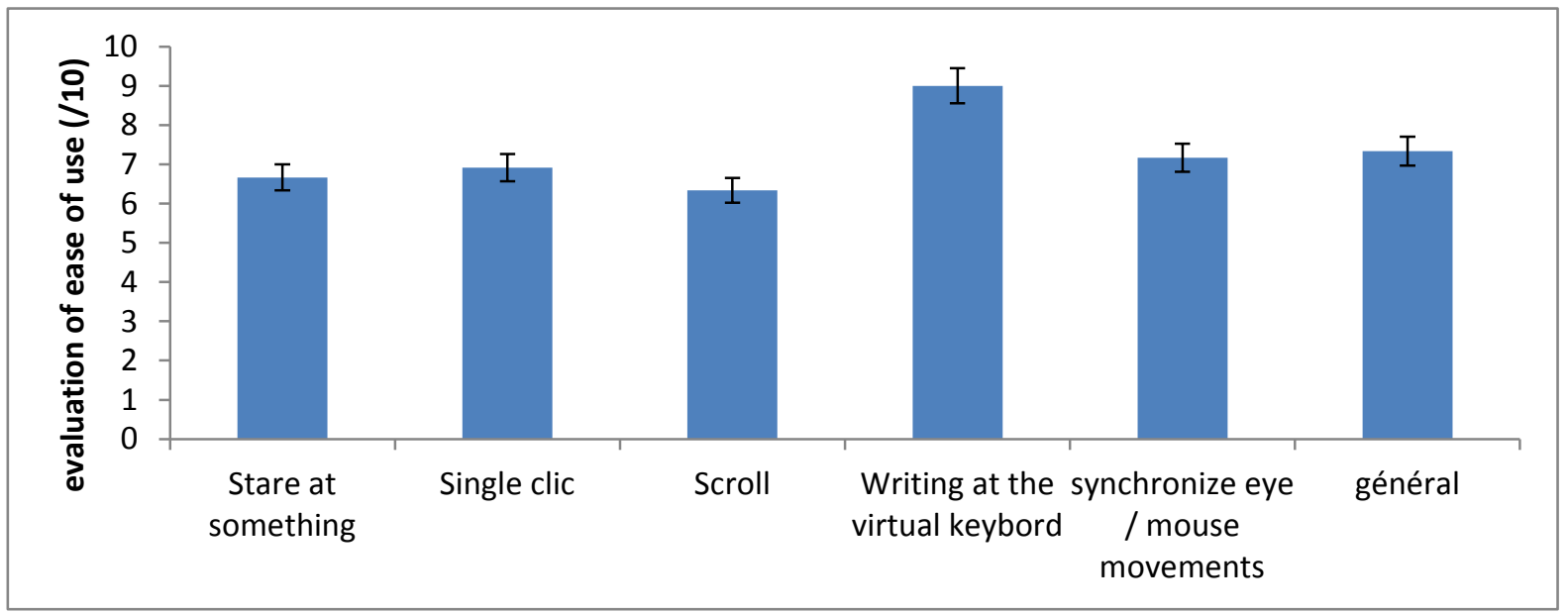

Figure 4 : ease of use scores for each interaction

\subsection{Discussion}

First, although only 12, the participants ( 9 men and 3 women) were large enough to obtain a representative sample. Indeed, as presented by Nielsen (1994), a test carried out on at least 8 users makes it possible to highlight $80 \%$ of usability problems. Overall, participants find the system easy to understand, the scores assessing understanding for each of the interactions being between 8 and 10 (/ 10). Regarding ease of use, discrepancies or difficulties in handling at the start of use disturbed the participants and their use. It is relevant to note that the keyboard writing obtained an average score of 9/10 in ease of use. The system generates eye strain during use, as showed on the long questionnaire scores. The survey required more effort from participants and therefore caused more visual fatigue. The major problem encountered during these experiments was the almost constant lag between the point set by the participant and the point of view appearing on the screen. It is concluded that the system is not yet ready and that simple disturbances (movement of the computer, movement of the participant) shift the calibration point. O'Brien (2009) points out that very dark eyes can disturb the eyecontrol in the differentiation between the pupil and the iris, which would result in slower eye tracking. The lack of precision of users is therefore normal, especially since the gaze is an extremely fast information processing 
method (1 / 5th of a second according to Carter, Aldridge, Page and Parker, 2010), which gives it the ability to move very quickly in order to react to each stimulus. Finally, as eye control is at the dawn of its development, it is not unusual to encounter some difficulties which will be corrected in future versions of the software. The participants were all generally enthusiastic and finding the system impressive, its future versions will be able to satisfy users even more.

\section{Bone conduction headset: future of communications?}

\subsection{State of the art}

Gribenski (1994) distinguishes two types of listening, which can be seen in the Figure 5, one that can be achieved by air (via the three areas of the ear - external, middle and internal) and the other by the bones.

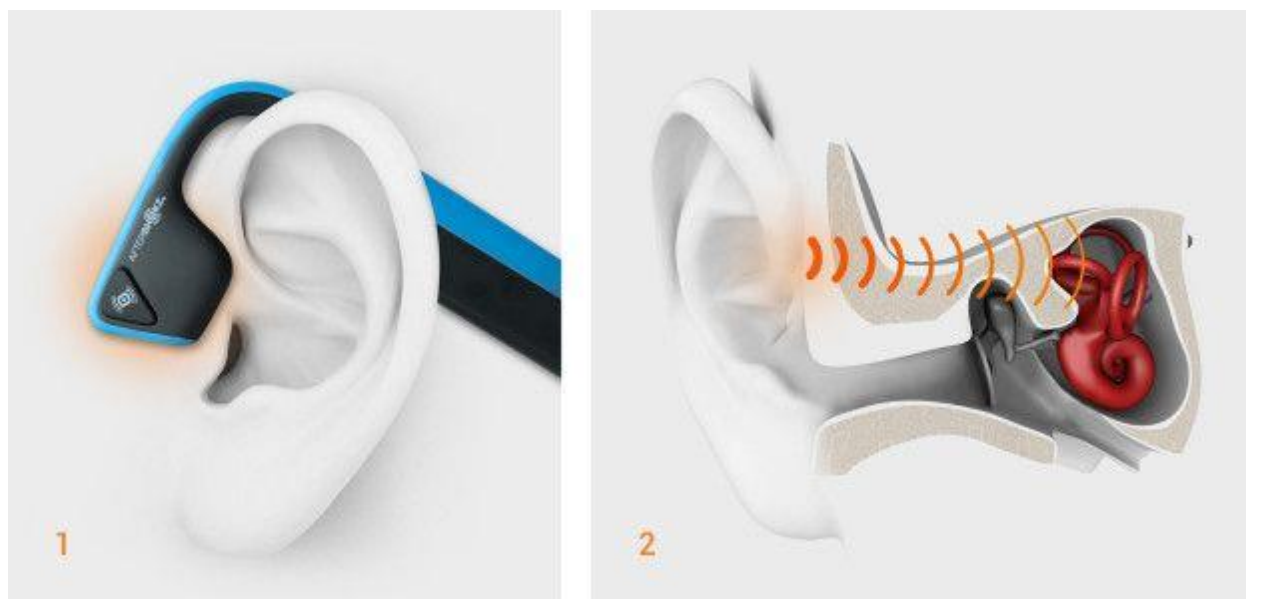

Figure 5 : illustration of the two types of listening (classic and bone conduction)

The waves are transmitted by the cerebral bone and arrive at the cochlea, in contact with this bone.

One of the first to use bone conduction was the famous musician Ludwig van Beethoven, who clasped a wooden rod between his teeth which he pressed on the piano body. For many years, researchers have been interested in osteophony, or bone conduction, designating the phenomenon of propagation of sound to the inner ear via the bones of the skull (Tonndorf, 1976). From the 1990s, bone conduction was miniaturized, developed for astronauts and the American Special Forces to communicate on the ground with free ears. It is therefore relevant to be interested in the impact of the bone conduction system for an application in the military naval field since this technology has already proven itself in the military field. Indeed, the use of a bone conduction headset can have an interest in situations requiring audio monitoring (from an external source such as a call or verbal information) while maintaining a perception of the environment Gripper Mcbride, Osafo-Yeboah \& Jiang (2007). However, this system also has its share of negative points. As shown by Gripper et al., (2007) and Manning, Mermagen and Scharine (2017), the bone conduction system did not show extraordinary performance (40dB less efficiency compared to conventional listening). Complex sounds like voice may appear distorted. There is also a question regarding the division of attention. Indeed, Hink, Van Voorhis, Hillyard and Smith (1977) describe humans as capable of processing different sound sources in parallel. However, they qualify this description by indicating that when the individual cannot be very attentive to a sound source in an acoustically disturbed environment. As individuals have a limited amount of attentional resources, their performance decreases sharply.

\subsection{Methods}

Participants were selected on a voluntary basis after responding to a questionnaire regarding their profile (age, gender and hearing problems). Twenty-four people participated in the experiment, including fifteen men and nine women. The average age of the participants was 36.5 years, the youngest was 23 , the oldest 60 . None of participants had hearing problems.

The equipment available to carry out this experiment was the bone conduction headset Aeropex Aftershokz, the step 2 of the NASA TLX questionnaire, and two computers to broadcast sounds (one in the environment and one in the headphones). 
After welcoming the participant, explaining the instructions and having him sign the consent, a short extract (30 seconds) of classical music is played in order to give a quick demonstration of how the system works. The experiment begins with a first session, with two tasks. During the first task, the participant listens on the one hand to seven animal sounds discontinuously (one sound every ten seconds) and must indicate whether the his corresponds to an animal image presented to him. On the other hand, a neutral report (chocolate cake) of one minute and fifteen seconds is broadcast in parallel. At the end, the participant must complete the part 2 of NASA TLX questionnaire. The second task is exactly similar to the first except that the report this time concerns the war, considered as a "committed" subject. The second session also includes two auditory sources and a recognition task but the two sources are continuous and in parallel. A warning alarm is given to the participant and he must press the space bar of the computer each time he hears the warning alarm among a soundtrack grouping together different alerts. In parallel with this task, the user hears, via the second auditory channel, a conversation between several people and must then answer questions relating to this discussion. The experiment then ends with a few questions, concerning the participant's feelings about the bone conduction headset, the positive and negative points he was able to identify and the cases of possible uses he could imagine for this technology in a naval defense context, the participants all working in the design of ships or associated services. Participants were divided into two groups; the experimental plan being represented on the Figure 6.

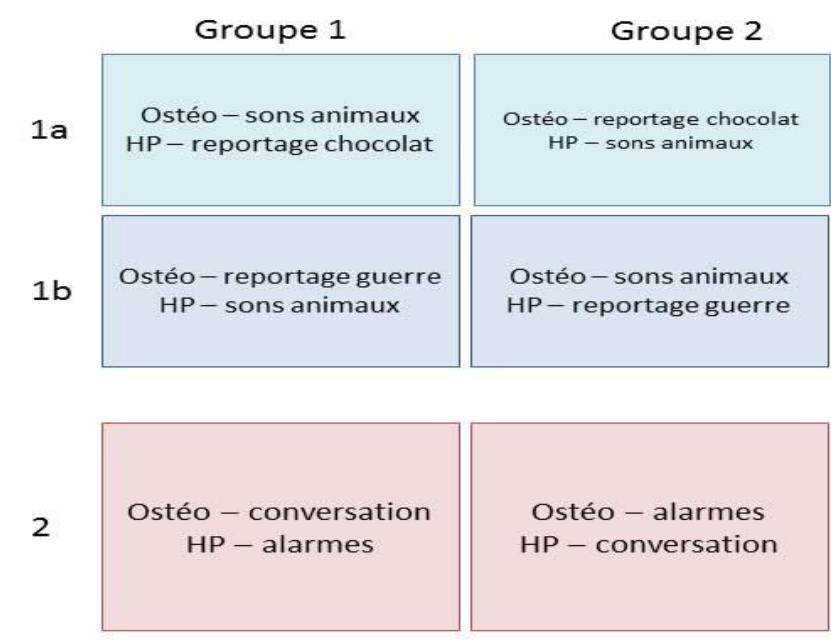

Figure 6 : experimental plan for bone conduction headset evaluation

\subsection{Results}

It is possible to observe on the Figure 7 that the scores change as the three different mental load assessments are made using the NASA TLX questionnaire. All the participants evaluated their performance score between 70 and 100 for the three parts (which is generally high and which induces a stable score). The increase is therefore linked to an increase in the perceived mental load or the perceived difficulty of the task (corresponding to the complexity of the tasks). The scores are relatively high, which indicates a perception of the burden by the participants, despite the lack of significance between the groups.

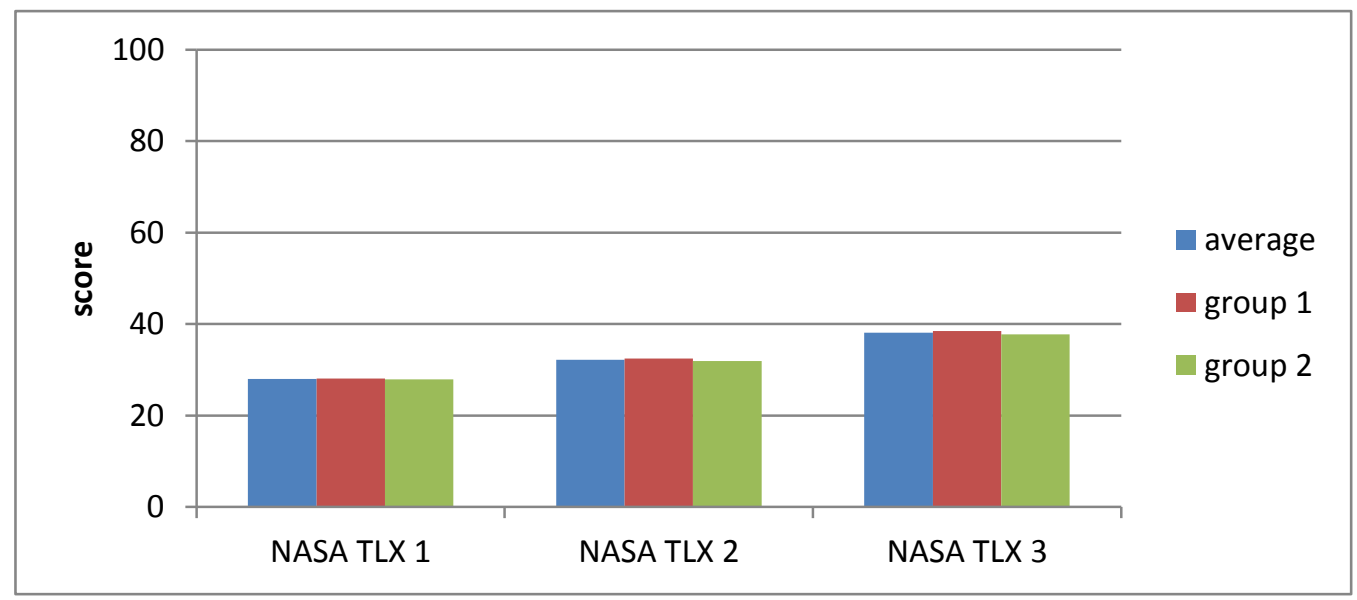

Figure 7 : increase of scores at the NASA TLX questionnaire 
There were no significant differences between the participants listening the bone conduction and those listening via the speakers, for the recognition of animals or comprehension of the reports. Furthermore, no significant difference was found between the different groups, genders or ages with regard to the recognition of alarms although participants who heard the alarms through the speakerphone respond a little faster than participants hearing the alarms through the bone conduction speaker. Participants were asked if they shared their attention between the two sources or if they focused on only one. Four of them said that they divided their attention on the two sources equally. Four others indicated, during the first two parts (the animals) to focus on the report and change their sound attention source as soon as the image appeared, serving as their visual guide. Six participants reported switching from one source to another at all times. Finally, twelve indicated that their attention was focused on one source, but that from time to time they spent their attention on the second for a short period of time.

\subsection{Discussion}

The results in terms of performance (answers to questions, validation of animals or alarms) lead us to say that participants can focus on both sources simultaneously, as had already been suggested in the work of Kahneman (1973) who presented the cognitive system as having a capacity of limited resources available at a time t. However, the more difficult the task, the more it will require attentive resources (Kahneman, 1973), in our case, the tasks comprising low technical or elaborate audio content, the participants are able to consciously process the main information of the reports in parallel alarm or animal type information. They were, however, unaware of this because, when the question was asked of them, they admitted that they were not paying attention to both sources. Thus, although they are not focused on the two sources in parallel, the information they perceive and process is sufficient to answer the questions. However, it is possible that by asking more specific questions, performance would have been lower. It is also necessary to consider the fact that if the different audio sources had been technical and of equal importance, the participant would not have been able to focus on the two in parallel and would have lost information.

The scores on the Nasa TLX questionnaire gradually increase during the experiment, for all participants, so it is possible to say that an increase in the workload takes place. However, it is not related to the visual task, this one allowing, according to the participants, to alleviate the difficulty of the task.

Despite the works of the literature suggesting that the human being is able to voluntarily prioritize one task over another, allowing to inhibit one of the two auditory sources (Hicks \& Tharpe, 2002; Crossley \& Hiscock, 1992; Pashler, 1994; Somberg \& Salthouse, 1982), nothing in the results allowed to conclude that the participants indicating that their attention is more focused on such and such a source are actually more efficient on the source in question.

\section{Throat microphone: an exploratory study}

\subsection{State of the art}

Throat microphones (Figure 8) are tools for capturing the vibrations of the vocal cords via the throat and transforming them into sound sources and thus replacing the sounds of "classical" speech transmitted thanks to the vibrations of air molecules in the mouth (Acker -Mills, Houtsma \& Ahroon, 2006). In the military context, operators can find themselves in situations that complicate communication and information sharing, like very noisy environments disturbing a standard acoustic microphone or environments requiring high discretion when speaking at a very low level.

The patent for the modern throat mic was filed by its inventor, Stuart Ballantine, an American, in 1935. This patent became public in June 1938. This system was present in armoured vehicles and aviation of army in 1940. The throat mic has been used, not widely, for many years in the military, and also by firefighters and some aircraft pilots. A parallel can be made with the naval field. Certain areas of ships can be noisy, in particular the flight deck of buildings or engine rooms, which can impair the vigilance and

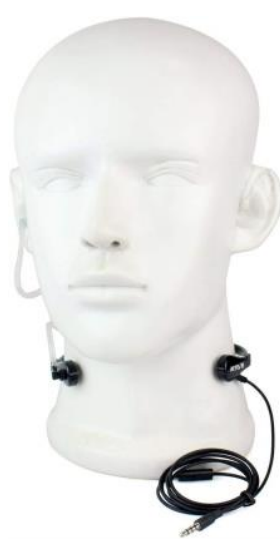

Figure 8 : a throat microphone performance (requires concentration efforts) of sailors (Grövel \& Stevanovic, 2017).

However, there are limits to the use of such technology as presented by Acker-Mills et al., (2006), who warned about the use of the throat mic. Indeed, few studies had been carried out on this date and they did not provide recommendations as to use. In addition, their study showed that throat mic causes poorer recognition of sounds than a normal microphone. The throat mic has shown performances which decrease significantly between $90 \mathrm{~dB}$ (63\% of correct recognition) and 106db (49\% of correct recognition) (Acker-Mills et al., 2006). The US military has conducted a study showing that the use of a laryngophone can affect speech intelligibility more than 
the noise generated by the use of an acoustic microphone (Barbara, 2004). In addition, it is necessary to pay attention to the placement of the system so that the capture of vibrations is done effectively (Ishak, 2017).

\subsection{Methods}

This study involves testing the laryngophone in order to identify its strengths and limitations from the point of view of its performance in terms of speech emission by the vibration of the vocal cords and the reception of these vibrations, and also to propose possible use cases in the military naval field. The evaluation of the laryngophone consisted of an evaluation centred on the use (installation, comfort, ...) of the laryngophone and the quality of reception (comprehension by the receiver) according to variable external conditions. The criterion of voice quality is a multidimensional phenomenon (Preminger and Van Tasell, 1995). The study was conducted by a human factors' specialist of Naval Group with three participants, two women and one man. Two criteria are taken for this evaluation:

- Loudness and intelligibility (level and comprehensibility of the speech signal, respectively) which allow the listener to hear and understand the speaker's message (between 2 laryngophones, between 1 laryngophone and 1 smartphone)

- Listening effort

\subsection{Results and interpretation}

First, the installation of the laryngophone is complex, with no indication of the location necessary for optimal operation, while the quality of the transmitted sound depends on the position of the electrodes on the neck. When the laryngophone is positioned in a "natural" way, it does not press very much on the throat and the sound is thus poorly transmitted. About the sound, it was impossible to assess between two laryngophones: strong feedback was present in one of the laryngophones. Also, for the sound to be better transmitted, it is then necessary for the user to manually hold the laryngophone at the top of the throat, under the tonsils and to press the electrodes if necessary so that the vibrations are well transmitted. This support on the throat is unpleasant and also causes discomfort to verbalize. The audio quality is thus dependent on the positioning of the laryngophone and also on the speaker's movements (turning the head, lowering the head, walking, etc.). This point was confirmed during Ishak's study (2017): at the slightest movement, this generates crackling and / or feedback in the device.

As expected on this type of device, no transfer of surrounding sounds is transmitted to the receiver. Only the voice is transmitted by vibration. Also, the type of voice does not seem to have an impact on the listening effort and the comprehension of the receiver. The type of content does not seem to have a strong impact on the listening effort and understanding of the receiver. It can however be noted that the short words are those which posed the most difficulties of understanding. This result is consistent with the models of oral comprehension: the listening process, active process of information processing, includes the phases of perception, decoding, and use (Anderson \& Lynch, 1988).

\section{General conclusions and opening}

In conclusion, despite the various limits, eye control and bone conduction headset were perceived as relevant, promising and fun, encouraging conclusions.

All the participants in these studies were involved and made many proposals for future use of the three systems.

For example, eye control can be used associated with mouse selection to select a track on operations MMI and activate some action on it. Or eye control can be also used to select some command not accessible by hand (when the distance between the system and the user is too far away).

For bone conduction, in operation room, it can be used to hear environment as well as communicate with someone. In some case, it is very important that the user doesn't be isolated to his environment (for example to hear order from his supervisor).

However, even if the conclusions were not as positive for the throat microphone, the experiment made it possible to rule on the usefulness and the usability of a system, which, once mature, could be relevant to test again.

These studies made it possible to evaluate innovative technology, to draw conclusions on their usability and to identify use cases on which to work for the implementation of this system on board. Although the results are encouraging for the implementation of these technologies (user satisfaction, numerous use cases and simple to set up according to operators), many points are still pending and can only be validated after studies more indepth with real future users, on board or in an operational situation, in order to ensure the proper functioning of 
the equipment (connectivity, efficiency) and the correct response to user needs, so as not to be exposed to a rejection of acceptability of this technology. These studies are the first step of this human centred approach.

Of course, this approach has to be applied with all new technologies (next year we want to investigate et assess for example gestural interaction). They are or will be evaluated through this Human factors approach mixed objective and subjective users performance measures to prove their efficiency for users before to be integrated in an operational system.

\section{References}

Acker-Mills, B. E., Houtsma, A. J., \& Ahroon, W. A. (2006). Speech intelligibility in noise using throat and acoustic microphones. Aviation, space, and environmental medicine, 77(1), 26-31.

Anderson, A., \& Lynch, T. (1988). Listening Oxford University Press. Call M (1985)‘Auditory Short Term Memory, Listening Comprehension, and the Input Hypothesis’ in TESOL QUARTERLY, 19, 765-779.

Barbara E. Acker-Mills, Adrianus J. Houtsma, William A. Ahroon, Speech Intelligibility in Noise Using Throat and Acoustic Microphones, USAARL (United States Army Aeromedical Research Laboratory), Aircrew Protection Division, Fort Rucker (Alabama), rapport 2004-13, avril 2004, 23 pages.

Carter, R., Aldridge, S., Page, M., \& Parker, S. (2010). Le grand Larousse du cerveau. Larousse.

Crossley, M., \& Hiscock, M. (1992). Age-related differences in concurrent-task performance of normal adults: evidence for a decline in processing resources. Psychology and aging, 7(4), 499.

Drewes, H. (2010). Eye gaze tracking for human computer interaction (Doctoral dissertation, lmu).

Gribenski, A. (1994). L'audition. FeniXX.

Gripper, M., McBride, M., Osafo-Yeboah, B., \& Jiang, X. (2007). Using the Callsign Acquisition Test (CAT) to compare the speech intelligibility of air versus bone conduction. International journal of industrial ergonomics, 37(7), 631-641.

Grövel, A., Stevanovic, J., \& Maruani, M. (2017). Travailler à bord des navires de la Marine marchande. Étude sociologique des risques et des violences physiques, psychologiques ou acaractere sexuel.

Hicks, C. B., \& Tharpe, A. M. (2002). Listening effort and fatigue in school-age children with and without hearing loss. Journal of Speech, Language, and Hearing Research.

Hink, R. F., Van Voorhis, S. T., Hillyard, S. A., \& Smith, T. S. (1977). The division of attention and the human auditory evoked potential. Neuropsychologia, 15(4-5), 597-605.

Ishak, D. (2017). La conception d'un système ultrasonore passif couche mince pour l'évaluation de l'état vibratoire des cordes vocales (Doctoral dissertation, Valenciennes).

Laharie A.M. - Intérêt des visiotests en médecine du travail. Paris, thèse de doctorat en médecine, 1982, 87 p.

Manning, C., Mermagen, T., \& Scharine, A. (2017). The effect of sensorineural hearing loss and tinnitus on speech recognition over air and bone conduction military communications headsets. Hearing research, $349,67-75$.

Nielsen, J., \& Mack, R. L. (Eds.). (1994). Usability inspection methods (Vol. 1). New York: Wiley.

O'Brien, S. (2009). Eye tracking in translation process research: methodological challenges and solutions. Methodology, technology and innovation in translation process research, 38, 251-266.

Ohno, T. (1998, July). Features of eye gaze interface for selection tasks. In Proceedings. 3rd Asia Pacific Computer Human Interaction (Cat. No. 98EX110) (pp. 176-181). IEEE.

Pashler, H. (1994). Dual-task interference in simple tasks: data and theory. Psychological bulletin, 116(2), 220.

Preminger, J. E., \& Tasell, D. J. V. (1995). Quantifying the relation between speech quality and speech intelligibility. Journal of Speech, Language, and Hearing Research, 38(3), 714-725.

Römer, T. (1939). Die Ruhelage der Augen und ihr Einfluß auf Beobachtungsabstand und visuelle Ermüdung bei Bildschirmarbeit*. Zeitschrift für experimentelle und angewandte psychologie, 36(4), 538-566. 
Rudall, B. H., \& Mann, C. J. H. (2007). New initiatives in building human-computer interfaces. Kybernetes, 36(1), 6-12.

Sibert, L. E., \& Jacob, R. J. (2000, April). Evaluation of eye gaze interaction. In Proceedings of the SIGCHI conference on Human Factors in Computing Systems (pp. 281-288). ACM.

Somberg, B. L., \& Salthouse, T. A. (1982). Divided attention abilities in young and old adults. Journal of Experimental Psychology: human perception and performance, 8(5), 651.

Tonndorf, J. (1976). Bone conduction. In Auditory System (pp. 37-84). Springer, Berlin, Heidelberg.

Vertegaal, R. (2008, October). A Fitts Law comparison of eye tracking and manual input in the selection of visual targets. In Proceedings of the 10th international conference on Multimodal interfaces (pp. 241248). ACM.

Zijlstra, F. R. H., \& Van Doorn, L. (1985). The construction of a scale to measure perceived effort. University of Technology. 